\title{
Active and passive behaviors of soft tissues: Pelvic floor muscles
}

\author{
M. P. M. Pato ${ }^{1,2, *, \dagger}$ and P. Areias ${ }^{2,3}$ \\ ${ }^{1}$ Departamento de Engenharia Química, Instituto Superior de Engenharia de Lisboa, Lisboa, Portugal \\ ${ }^{2}$ ICIST_Departamento de Engenharia Civil, Instituto Superior Técnico \\ ${ }^{3}$ Departamento de Física, Universidade de Évora, Évora, Portugal
}

\begin{abstract}
SUMMARY
A new active-contraction visco-elastic numerical model of the pelvic floor (skeletal) muscle is presented. Our model includes all elements that represent the muscle constitutive behavior, contraction and relaxation. In contrast with the previous models, the activation function can be null. The complete equations are shown and exactly linearized. Small verification and validation tests are performed and the pelvis is modeled using the data from the intra-abdominal pressure tests. Copyright $\mathbb{C} 2009$ John Wiley \& Sons, Ltd.
\end{abstract}

Received 30 March 2009; Revised 17 August 2009; Accepted 13 September 2009

KEY WORDS: pelvic floor skeletal muscle; active behavior; shell model; geometrically nonlinear

\section{In memory of Professor J. A. C. Martins}

\section{INTRODUCTION}

Pelvic floor muscles are neuronally controlled and held together by connective tissue arranged in a unique three-dimensional (3D) arrangement. Together, the structure formed of three classes of tissue affects pelvic organ support and function. They are tonically active in all positions and contract phasically in reaction to bladder filling and intra-abdominal pressure (IAP), whereas activity ceases with micturition. In this study we develop a biomechanical model of skeletal muscles when the activation falls with no excitation.

From the mechanical viewpoint, the material behavior of muscles is highly nonlinear. They undergo large deformations, changing shape significantly. However, models of this kind require realistic constitutive relations between stress and strain measures that are difficult to determine in an inhomogeneous material. Since data measuring is intricate, simulations are hard to validate. We ensure that the mathematical problem is well-posed with numerical verification.

The present approach is based on the use of the finite element method (FEM). The material behavior of the muscle is split into an active (representing the movement of the actin and myosin filaments-the muscle contraction) and a passive part (representing the connective tissue in series with the sarcomeres and the parallel connective tissue that surround the contractile element). To describe the passive part, special unit cells consist of one triangular shell element (S3R in ABAQUS). The passive properties can be described by nonlinear hyperelastic and incompressible constitutive relations [1]; moreover, because of the presence of a single muscle fiber direction they may be considered as transversely isotropic.

\footnotetext{
${ }^{*}$ Correspondence to: M. P. M. Pato, Departamento de Engenharia Química, Instituto Superior de Engenharia de Lisboa, Lisboa, Portugal.

†E-mail: mpato@civil.ist.utl.pt, mpato@deq.isel.ipl.pt 
The first mathematical model was created by Archibald Vivian Hill [1-3] and was onedimensional (1D). It was composed of three elements: a (active) contractile element in series with an (passive) elastic element, both of them in parallel with a third (passive) elastic element. This phenomenological Hill-type model accounts for the force-length-velocity property of muscle and can be used to calculate the muscle force if values of muscle length, velocity, and muscle activation are known. The contractile element is responsible for the free change in length of the muscle (when not activated) and for the force production in the muscle (when activated). The model discussed here is derived from the inaugural Hill model.

These types of models are used in the movement analysis and muscle performance studies of multibody dynamics [3-16] in which current medical imaging capabilities, segmentation and meshing software have been used to capture and model the 3D geometry and movement of skeletal muscles. Recently, Van Loocke et al. [17] provided experimental data on the compression behavior of skeletal muscles along the fibers and transversely, and it was compared with those obtained by Grieve and Armstrong [18] and Bosbbom et al. [19].

In some cases, the evaluation of the unknown kinematic, force, activation and neural stimulation variables in a time interval involves forward dynamic calculations and requires the resolution of optimal control problems. An important property of the discrete models proposed by Otten and Hulliger [20] and van Leeuwen and Kier [21] is the separation of the tissue in a fiber-solid part and a fluid part. The solid part is described by hydrostatic pressure. In a continuum model, both aspects are interrelated. His model includes hydrostatic pressure as a property of the whole tissue and requires volume conservation as a geometrical constraint.

The Hill model can be used to calculate the generated force from electromyography (EMG) measurements at the middle of the muscle. Assuming that each muscle unit produces the same force, the total muscle force is the sum of all parallel units in each muscle.

\section{GOVERNING EQUATIONS}

The constitutive equation adopted in this work for 3D skeletal muscles includes both their passive and active behaviors. Here we present a modified form of the incompressible transversely isotropic hyperelastic model proposed by Humphrey et al. [22] for passive cardiac tissues. Similar to the skeletal muscles, the anisotropy of the passive cardiac model follows the criterion of Spencer [23] for fiber-reinforced incompressible hyperelastic composites. The strain energy density function follows the Humphrey model, which corresponds to the sum of the terms of stress fiber and embedding matrix, assumed to be isotropic. The modifications we make here ensure compatibility between the passive and active longitudinal behaviors of the skeletal muscles in tension. They are consistent with the available experimental results on passive compression of skeletal muscles presented by Grieve and Armstrong [18]. This is consistent with the 1D models of skeletal muscles proposed by the Hill model.

The constitutive equation for the Cauchy stress tensor $\sigma$ has the contributions of the incompressibility or quasi incompressibility of the muscle, and an assumed embedding matrix as well as the longitudinal (tension) behavior of the muscle fibers:

$$
\boldsymbol{\sigma}=\boldsymbol{\sigma}_{\text {incomp }}+\boldsymbol{\sigma}_{\text {matrix }}+\boldsymbol{\sigma}_{\text {fiber }}
$$

The first contribution, in the case of the perfect incompressible $(J=\operatorname{det} \mathbf{F}=1)$, has the form

$$
\boldsymbol{\sigma}_{\text {incomp }}=-p \mathbf{I}
$$

where $p$ is the hydrostatic pressure and $\mathbf{I}$ is the second-order identity tensor; in the case of quasi incompressible it has the following form:

$$
\boldsymbol{\sigma}_{\text {incomp }}=\frac{1}{D} \frac{\mathrm{d} U_{J}}{\mathrm{~d} J} \mathbf{I}
$$

where $D$ is the compressibility compliance and $U_{J}$ is an energy function that grows with $J$ in order to penalize the volume change, and grows unbounded as $J \rightarrow 0$, to prevent material collapse. 
Before specifying the constitutive laws for $\boldsymbol{\sigma}_{\text {matrix }}$ and $\boldsymbol{\sigma}_{\text {fiber }}$, the conditions $\sigma_{i_{t}}=0, i_{t} \in\{1,2,3\}$, must be introduced; it is sufficient to specify $p$. The condition $\sigma_{33}=0$ imposes a particular form for the pressure, which is easily shown to be

$$
p=\frac{1}{3}\left[2 U_{I}^{\prime}\left(\operatorname{tr}\left[\mathbf{b}_{p}\right]-2 C_{33}\right)+\lambda_{f} U_{f}^{\prime}\left(\mathbf{n}_{p} \otimes \mathbf{n}_{p}\right)\right]
$$

The deformation gradient is decomposed as

$$
\mathbf{F}=\left[\begin{array}{cc}
\mathbf{F}_{p} & 0 \\
0 & F_{33}
\end{array}\right]
$$

with

$$
F_{33}=\operatorname{det}\left[\mathbf{F}_{p}\right]^{-1}
$$

The left Cauchy-Green tensor, $\mathbf{b}$, is given by

$$
\mathbf{b}=\left[\begin{array}{cc}
\underbrace{\mathbf{F}_{p} \mathbf{F}_{p}^{\mathrm{T}}}_{\mathbf{b}_{p}} & 0 \\
0 & F_{33}^{2}
\end{array}\right]
$$

and the right Cauchy-Green tensor, $\mathbf{C}$, follows its definition:

$$
\mathbf{C}=\left[\begin{array}{cc}
\underbrace{\mathbf{F}_{p}^{\mathrm{T}} \mathbf{F}_{p}}_{\mathbf{C}_{p}} & 0 \\
0 & F_{33}^{2}
\end{array}\right]
$$

\section{Remark 1}

Antman and Schuricht [24] provide a more complete derivation for the incompressibility problem in a shell. Ours is a convenient method appropriate for the present application.

The matrix contribution is isotropic hyperelastic, similar to those adopted in other soft tissues; the specific form is

$$
\boldsymbol{\sigma}_{\text {matrix }}=2 b c \exp \left[b\left(I_{1}^{C}-3\right)\right] \operatorname{dev}\left[\mathbf{b}_{p}\right]
$$

where $b$ and $c$ are constitutive constants, $\mathbf{b}_{p}$ is the left Cauchy-Green strain tensor and $\operatorname{dev}[\bullet]=$ $(\bullet)-\frac{1}{3} \operatorname{tr}[\bullet] \mathbf{I}$ with $\bullet \in$ Lin being the deviatoric operator in the spatial configuration (a projection linear transformation).

The Cauchy stress contribution of the muscle fibers has the form

$$
\boldsymbol{\sigma}_{\text {fiber }}=\operatorname{dev}\left[\lambda_{f} T \mathbf{n}_{p} \otimes \mathbf{n}_{p}\right]
$$

where it is assumed that the muscle is incompressible. The scalar $T$ represents the nominal stress in the fiber, the force per unit area of the cross-section of its reference configuration, and the current muscle fiber vector $\mathbf{n}_{p}$ has the form:

$$
\mathbf{n}_{p}=\frac{\mathbf{F}_{p} \mathbf{N}_{p}}{\lambda_{f}}
$$

with $\lambda_{f}$ being the stretch ratio of the muscle fibers, which have the direction of the unit vector $\mathbf{N}_{p}$ in the undeformed configuration given by

$$
\lambda_{f}=\sqrt{\mathbf{N}_{p}^{\mathrm{T}} \mathbf{C}_{p} \mathbf{N}_{p}}
$$


Remark 2

Consider $\lambda_{f}=L / L_{0}^{M}$ in the reference configuration with direction $\mathbf{N}$ and an elementar length $\mathrm{d} s_{0}$, whereas in the current configuration we have the direction $\mathbf{N}$ and an elementar length $\mathrm{d} s$, then $\mathrm{d} s \mathbf{n}=\mathbf{F d} s_{0} \mathbf{N} \longleftrightarrow \lambda_{f} \mathbf{n}=\mathbf{F N}$ and $\lambda_{f}^{2} \mathbf{n . n}=(\mathbf{F N}) \cdot(\mathbf{F N})$.

The longitudinal muscle stress $T$ is additively decomposed as

$$
T=T^{\mathrm{PE}}+T^{\mathrm{SE}}
$$

where $T^{\mathrm{PE}}$ and $T^{\mathrm{SE}}$ are the parallel and series elements.

The stress $T^{\mathrm{CE}}$ in the contractile element equals the stress in the series elastic element:

$$
T^{\mathrm{CE}}=T^{\mathrm{SE}}
$$

and must be given in general by the product of

1. A function of the contractile stretch with maximum value at the muscle rest length.

2. A function of the strain rate of the contractile element, which corresponds, for the contracting rates, to Hill's hyperbolic law.

3. An activation variable $\alpha \in\left[\alpha_{\min }, 1\right]$ with $\alpha_{\min } \geqslant 0$.

We make use of the multiplicative stretch split of the fiber stretch $\left(\lambda_{f}\right)$ into a contractile stretch $\left(\lambda^{\mathrm{CE}}\right)$ and an elastic stretch $\left(\lambda^{\mathrm{SE}}\right)$ :

$$
\lambda_{f}=\lambda^{\mathrm{CE}} \lambda^{\mathrm{SE}}
$$

This corresponds to an additive Hencky strain decomposition.

The stress $T^{\mathrm{PE}}$ in the parallel elastic element is a product of the maximum muscle stress at rest (at rest, the muscle can withstand stresses up to $T_{0}^{\mathrm{M}}$ but no more):

$$
T^{\mathrm{PE}}\left(\lambda_{f}\right)=T_{0}^{\mathrm{M}} f^{\mathrm{PE}}\left(\lambda_{f}\right)
$$

where

$$
f^{\mathrm{PE}}\left(\lambda_{f}\right)= \begin{cases}2 a A \exp \left[a\left(\lambda_{f}-1\right)^{2}\right]\left(\lambda_{f}-1\right), & \lambda_{f}>1 \\ 0 & \text { otherwise }\end{cases}
$$

which is a rederived version of the proposal by Pandy et al. [3]. Constitutive parameters $a$ and $A$ were introduced, for cardiac tissue, by Humphrey and Yin [25]. The stress $T^{\mathrm{SE}}$ in the series element is given by an analogous decomposition

$$
T^{\mathrm{SE}}\left(\lambda^{\mathrm{SE}}, \lambda^{\mathrm{CE}}\right)=T_{0}^{M} f^{\mathrm{SE}}\left(\lambda^{\mathrm{SE}}, \lambda^{\mathrm{CE}}\right)
$$

with

$$
f^{\mathrm{SE}}\left(\lambda_{f}, \lambda^{\mathrm{CE}}\right)=\frac{1}{10}\left\{\exp \left[100\left(\lambda_{f}-\lambda^{\mathrm{CE}}\right)\right]-1\right\}, \quad \lambda_{f} \geqslant \lambda^{\mathrm{CE}}
$$

This expression was obtained by rederiving Pandy et al. [3] equations. The functions $T^{\mathrm{PE}}\left(\lambda_{f}\right)$ and $T^{\mathrm{SE}}\left(\lambda^{\mathrm{SE}}, \lambda^{\mathrm{CE}}\right)$ are strictly positive. A representation of the two non-dimensional forces and their sum is shown in Figure 1.

The $T^{\mathrm{CE}}$ stress follows the relatively standard form, adapted from Pandy et al. [3]:

$$
T^{\mathrm{CE}}\left(\lambda^{\mathrm{CE}}, \dot{\lambda}^{\mathrm{CE}}, \alpha\right)=T_{0}^{M} f_{L}^{\mathrm{CE}}\left(\lambda^{\mathrm{CE}}\right) f_{V}^{\mathrm{CE}}\left(\dot{\lambda}^{\mathrm{CE}}\right) \alpha
$$

where

$$
f_{L}^{\mathrm{CE}}\left(\lambda^{\mathrm{CE}}\right)=8 \begin{cases}\lambda^{\mathrm{CE}^{2}}-\lambda^{\mathrm{CE}}+0.25, & 0.5 \leqslant \lambda^{\mathrm{CE}}<0.75 \\ -\lambda^{\mathrm{CE}}+2 \lambda^{\mathrm{CE}}-0.875, & 0.75 \leqslant \lambda^{\mathrm{CE}}<1.25 \\ \lambda^{\mathrm{CE}^{2}-3 \lambda^{\mathrm{CE}}+2.25,} & 1.25 \leqslant \lambda^{\mathrm{CE}}<1.5 \\ 0 & \text { otherwise }\end{cases}
$$




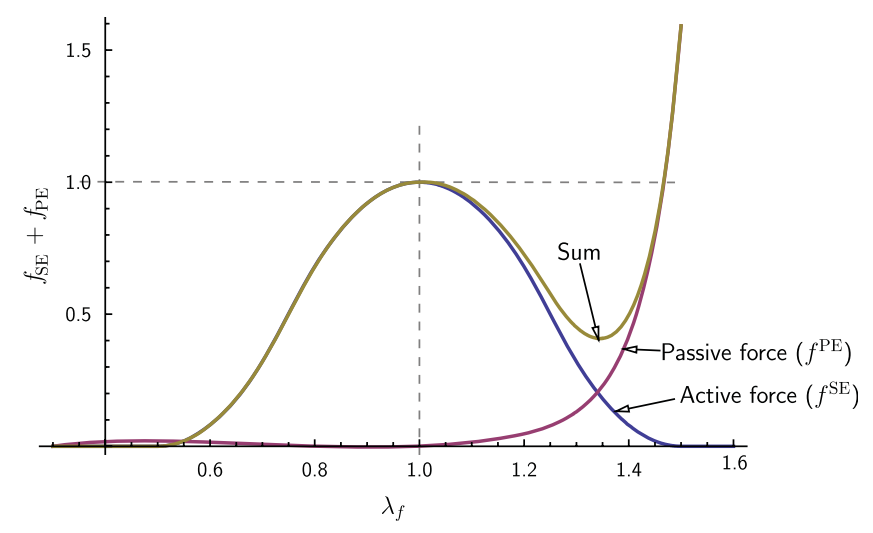

Figure 1. Passive $\left(f^{\mathrm{PE}}\right)$ and active $\left(f^{\mathrm{SE}}\right)$ non-dimensional forces.

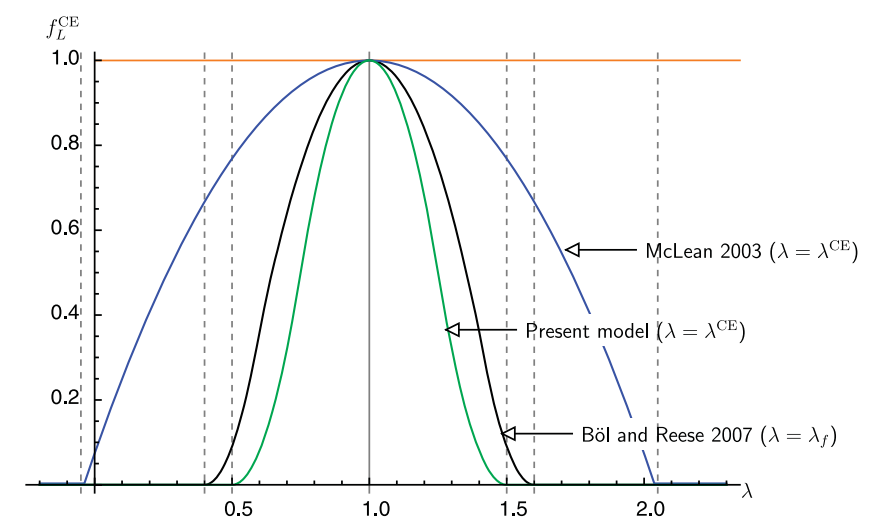

Figure 2. Representation of $f_{L}^{\mathrm{CE}}$ for our model and references [26, 27].

and

$$
f_{V}^{\mathrm{CE}}\left(\dot{\lambda}^{\mathrm{CE}}\right)= \begin{cases}\left.\dot{\lambda}^{\mathrm{CE}}+10\right) \varepsilon, & \dot{\lambda}^{\mathrm{CE}} \leqslant-10 \mathrm{~s}^{-1} \\ -\frac{\arctan \left(-0.5 \dot{\lambda}^{\mathrm{CE}}\right)}{\arctan (5)}+1, & -10 \mathrm{~s}^{-1}<\dot{\lambda}^{\mathrm{CE}}<2 \mathrm{~s}^{-1} \\ \left.\dot{\lambda}^{\mathrm{CE}}-2\right) \varepsilon+\frac{\pi}{4 \arctan (5)}+1, & \dot{\lambda}^{\mathrm{CE}} \geqslant 2 \mathrm{~s}^{-1}\end{cases}
$$

The functions $f_{L}^{\mathrm{CE}}$ and $f_{V}^{\mathrm{CE}}$ are proposed here for the first time and avoid finding the inverse functions. The value of $\varepsilon$ is sufficiently small, and is introduced to avoid $f_{V}^{\mathrm{CE}}=0$ when $\dot{\lambda}^{\mathrm{CE}} \leqslant-10 \mathrm{~s}^{-1}$. The specific form (19) is introduced in this work as the definite remedy for the well-known discontinuities. Alternatives to this function were provided recently by Böl and Reese [26] and Mclean et al. [27]. The functions $f_{L}^{\mathrm{CE}}$ and $f_{V}^{\mathrm{CE}}$ map $\left.\left(\lambda^{\mathrm{CE}}, \dot{\lambda}^{\mathrm{CE}}\right) \in\right] 0,+\infty\left[\times \mathbb{R}\right.$ into $T^{\mathrm{CE}} \in \mathbb{R}$. A comparison between the three models can be seen in Figure 2.

Our non-smooth $f_{V}^{\mathrm{CE}}$ function is represented in Figure 3. We can note the following:

1. Böl and Reese use $\lambda_{f}$ and $\dot{\lambda}_{f}$ replacing $\lambda^{\mathrm{CE}}$ and $\dot{\lambda}^{\mathrm{CE}}$, respectively. This simplification is required to circumvent the singularity problem. We solved the singularity problem and therefore can do without this simplification.

2. Our model, in contrast with all other we have seen in the literature, allows $u=0$ (null neural stimulus) with no singularity problems. 


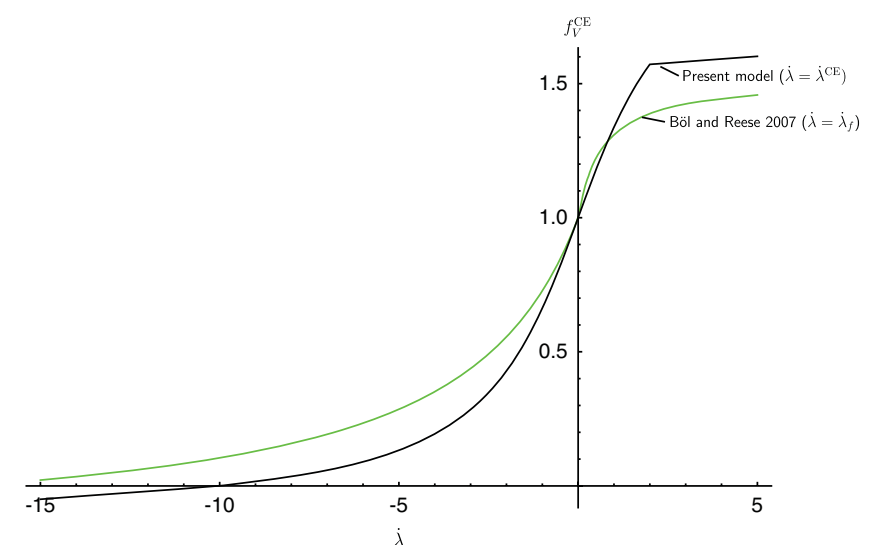

Figure 3. Representation of $f_{V}^{\mathrm{CE}}$ for our model and Reference [26].

3. In contrast with the model by Böl and Reese (see Table III in [26]), our model requires no parameters.

The time-dependent activation process involves the contractile element and is caused by neural excitation. It is represented at the macroscopic level by the first-order ordinary differential equation (ODE) and follows Pandy et al. [3]:

$$
\dot{\alpha}(t)=\frac{1}{\tau_{\text {rise }}}(1-\alpha(t)) u(t)+\frac{1}{\tau_{\text {fall }}}\left(\alpha_{\min }-\alpha(t)\right)(1-u(t))
$$

Remark 3

The contraction process is controlled neurologically, not all muscles fibers are simultaneously excited. However, we consider that hypothesis as valid.

\section{Remark 4}

The total contraction muscle force depends on the number of excited fibers.

\section{Remark 5}

Muscle activation appears as an intermediate variable integrating the sequence of neural discharge and describing the level of contraction. An increase of tension implies an increase in the stimulation rate.

\section{Remark 6}

In an isometric test, the tension depends only on the activation function, since $\lambda_{f}=\lambda^{\mathrm{CE}}$ and $f_{L}^{\mathrm{CE}} f_{V}^{\mathrm{CE}}=1$.

In this equation the $\tau_{\text {rise }}$ and $\tau_{\text {fall }}$ are characteristic time constants for activation and deactivation of the muscle, and $\alpha_{\min }$ is the minimum value of activation. The function $u(t)$ ranges from 0 to 1 , and represents the neural excitation, and is the input data for the model. The activation $\alpha(t)$ ranges from $\alpha_{\min } \geqslant 0$ to 1 . The time-dependent inputs are the normalized activation level of the muscles $(u)$ and the muscle length changes $\left(\lambda^{\mathrm{PE}}\right)$.

The present constitutive behavior can be written by

$$
\boldsymbol{\sigma}=-p \mathbf{I}+2 \operatorname{dev}\left[\mathbf{F} \frac{\partial U}{\partial \mathbf{C}} \mathbf{F}^{\mathrm{T}}\right]
$$

which represents the Cauchy stress.

The incompressibility constraint is imposed, and the time rate of change of the internal variable $\lambda^{\mathrm{CE}}$ is governed by a differential inclusion of the type

$$
\dot{\lambda}^{\mathrm{CE}} \in \mathscr{L}^{\mathrm{CE}}\left(\lambda_{f}, \lambda^{\mathrm{CE}}, \alpha, \dot{\lambda}_{f}, u\right)
$$


The other internal variable is controllable by the mentioned differential equation of $\dot{\alpha}(u, t)$. In terms of strain energy $U$ is given by

$$
U=U\left(\mathbf{C}, \lambda^{\mathrm{CE}}\right)=U_{\text {matrix }}\left(I_{1}^{C}\right)+U^{\mathrm{PE}}\left(\lambda_{f}\right)+U^{\mathrm{SE}}\left(\frac{\lambda_{f}}{\lambda^{\mathrm{CE}}}, \lambda^{\mathrm{CE}}\right)
$$

\section{INTEGRATION}

To solve the constitutive problem stated before, we need to obtain the value of $\lambda^{\mathrm{CE}}$ in all study cases, like isometric, isotonic and quick-release in different situations: with or without neural excitation, and/or with and without load:

$$
\begin{aligned}
\dot{\lambda}^{\mathrm{CE}}\left(\lambda_{f}, \lambda^{\mathrm{CE}}, \alpha, \dot{\lambda}_{f}, u\right) & =f_{V}^{\mathrm{CE}^{-1}}\left(\lambda_{f}, \lambda^{\mathrm{CE}}, \alpha, \dot{\lambda}_{f}, u\right) \\
\dot{\alpha}(u, t) & =\frac{1}{\tau_{\text {rise }}}(1-\alpha(t)) u(t)+\frac{1}{\tau_{\text {fall }}}\left(\alpha_{\min }-\alpha(t)\right)(1-u(t))
\end{aligned}
$$

The method used is backward Euler (implicit and unconditionally stable) for the two differential equations above mentioned, with initial conditions. These equations are

$$
\begin{aligned}
\frac{\partial \dot{\lambda}^{\mathrm{CE}}}{\partial \lambda^{\mathrm{CE}}} & =\left(\frac{\partial f_{V}^{\mathrm{CE}}}{\partial \lambda^{\mathrm{CE}}}\right)^{-1}\left(\lambda_{f}, \lambda^{\mathrm{CE}}, \alpha, \dot{\lambda}_{f}, u\right) \\
& = \begin{cases}\frac{1}{\varepsilon} \frac{\partial f_{V}^{\mathrm{CE}}}{\partial \lambda^{\mathrm{CE}}}, & \frac{\pi}{4 \arctan (5)+1}<f_{V}^{\mathrm{CE}}<0 \\
\frac{2 \arctan (5) \frac{\partial f_{V}^{\mathrm{CE}}}{\partial \lambda^{\mathrm{CE}}}}{\cos ^{2}\left(\arctan (5)\left(f_{V}^{\mathrm{CE}}-1\right)\right)} & \text { otherwise }\end{cases}
\end{aligned}
$$

and with $\lambda^{\mathrm{CE}}(0)=0.9959455676, \alpha(0)=u(0)=0$. Also, $\lambda_{f}(0)=1$. The value of $\lambda^{\mathrm{CE}}(0)$ is obtained by the following system:

$$
\begin{aligned}
f_{V}^{\mathrm{CE}} f_{L}^{\mathrm{CE}}\left(\lambda^{\mathrm{CE}}\right) \alpha & =f^{\mathrm{SE}}\left(\lambda_{f}, \lambda^{\mathrm{CE}}\right) \\
f_{V}^{\mathrm{CE}} \alpha & =1 \\
\lambda_{f} & =1
\end{aligned}
$$

without deformation. The constants are taken from Humphrey and Yin [25], for incompressible isotropic cardiac tissue (see Table I). Note that our $\alpha_{\min }$ is zero because no singularity occurs. The ratio between the time constants is usually $\tau_{\text {rise }} / \tau_{\text {fall }}=0.1$.

Table I. Material properties for the cardiac tissue.

\begin{tabular}{lc}
\hline Variable & Value \\
\hline$a$ & 12.43 \\
$b$ & 23.46 \\
$c$ & $3.79517355 \times 10^{-4} \mathrm{MPa}$ \\
$A$ & $5.7270836 \times 10^{-4} \mathrm{MPa}$ \\
$T_{0}^{M}$ & $6.5586872 \times 10^{1} \mathrm{MPa}$ \\
$\tau_{\text {rise }}$ & $20 \times 10^{-3} \mathrm{~s}$ \\
$\tau_{\text {fall }}$ & $200 \times 10^{-3} \mathrm{~s}$ \\
$\alpha_{\text {min }}$ & 0 \\
\hline
\end{tabular}


As we see in Equation (23), to find the root we have used the trust region method. All equations mentioned above are implemented in a module of the subroutine UMAT from ABAQUS. There are three dependent state variables: $\lambda_{f}, \lambda^{\mathrm{CE}}$ and $\alpha$.

\section{CONSISTENT LINEARIZATION}

The reduced Cauchy stress tensor is calculated using the following constitutive formula:

$$
\boldsymbol{\sigma}_{p}=2 U_{I}^{\prime}\left(\mathbf{b}_{p}-b_{33} \mathbf{I}_{p}\right)+\lambda_{f} U_{f}^{\prime}\left(\mathbf{n}_{p} \otimes \mathbf{n}_{p}\right)
$$

The tangent modulus (consisting of the derivative of $\boldsymbol{\sigma}_{p}$ with respect to $\dot{\boldsymbol{\varepsilon}}$ ) is required to calculate the stiffness matrix:

$$
C_{i_{s} j_{s} k_{s} l_{s}}=\frac{1}{2}\left(\frac{\partial \sigma_{p_{i_{s} j_{s}}}}{\partial F_{k_{s} l_{s}}} F_{r_{s} l_{s}}+\frac{\partial \sigma_{p_{i_{s} j_{s}}}}{\partial F_{r_{s} l_{s}}} F_{k_{s} l_{s}}\right)
$$

Observing (29) we conclude that the derivatives of $U_{I}^{\prime}, \mathbf{b}_{p}, B_{33}, \lambda_{f}$ and $U_{f}^{\prime}$ are required to determine the tangent modulus. The corresponding derivatives of $\mathbf{n}_{p}$ are filtered by Abaqus, due to minor-symmetry. The calculation of these derivatives is straightforward and was performed with Mathematica 6.0 software. The only exception is the one for $U_{f}^{\prime}$ that requires the derivative with respect to $\lambda^{\mathrm{CE}}$, obtainable by using the implicit function theorem. We, therefore, present the details of this calculation.

After integration, the residual of contractile element stretch follows the backward-Euler form:

$$
r_{\lambda}=\lambda^{\mathrm{CE}}-\lambda_{\text {old }}^{\mathrm{CE}}-\Delta t \dot{\lambda}^{\mathrm{CE}}\left(\lambda_{f}, \lambda^{\mathrm{CE}}\right)
$$

from which (when the solution holds, $r_{\lambda} \approx 0$ ) the derivative of $\lambda^{\mathrm{CE}}$ with respect to $\mathbf{F}$ is obtained by implicit differentiation:

$$
\frac{\partial \lambda^{\mathrm{CE}}}{\partial \mathbf{F}}=\left(\frac{\Delta t \frac{\partial \dot{\lambda}^{\mathrm{CE}}}{\partial \lambda_{\mathrm{f}}}}{1-\Delta t \frac{\partial \dot{\lambda}^{\mathrm{CE}}}{\partial \lambda^{\mathrm{CE}}}}\right) \frac{\partial \lambda_{f}}{\partial \mathbf{F}} \approx 0
$$

We can directly obtain the derivative of $U_{f}^{\prime}$ as

$$
\begin{aligned}
\frac{\partial U_{f}^{\prime}}{\partial \mathbf{F}} & =\frac{\partial F^{\mathrm{PE}}}{\partial \lambda_{f}} \frac{\partial \lambda_{f}}{\partial \mathbf{F}}+\underbrace{\frac{\partial F^{\mathrm{SE}}}{\partial \lambda_{f}} \frac{\partial \lambda_{f}}{\partial \mathbf{F}}+\frac{\partial F^{\mathrm{SE}}}{\partial \lambda^{\mathrm{CE}}} \frac{\partial \lambda^{\mathrm{CE}}}{\partial \mathbf{F}}}_{\mathrm{d} F^{\mathrm{SE}} / \mathrm{d} \mathbf{F}} \\
& =\left[\frac{\partial F^{\mathrm{PE}}}{\partial \lambda_{f}}+\frac{\partial F^{\mathrm{SE}}}{\partial \lambda_{f}}+\frac{\partial F^{\mathrm{SE}}}{\partial \lambda^{\mathrm{CE}}}\left(\frac{\Delta t \frac{\partial \dot{\lambda}^{\mathrm{CE}}}{\partial \lambda_{f}}}{1-\Delta t \frac{\partial \dot{\lambda}^{\mathrm{CE}}}{\partial \lambda^{\mathrm{CE}}}}\right)\right] \frac{\partial \lambda_{f}}{\partial \mathbf{F}}
\end{aligned}
$$

In (32), the derivative of $F^{\mathrm{PE}}$ with respect to $\lambda_{f}$ is straightforward and therefore all quantities are easily calculated.

The derivative of $\boldsymbol{\sigma}_{p}$ with respect to $\mathbf{F}_{p}$ follows the chain rule

$$
\begin{aligned}
\frac{\partial \boldsymbol{\sigma}_{p}}{\partial \mathbf{F}_{p}}= & 2\left(\mathbf{b}_{p}-b_{33} \mathbf{I}_{p}\right) \otimes\left(\frac{\partial U_{I}^{\prime}}{\partial \mathbf{F}_{p}}+\frac{\partial U_{I}^{\prime}}{\partial b_{33}} \frac{\partial b_{33}}{\partial \mathbf{F}_{p}}\right)+2 U_{I}^{\prime}\left(\frac{\partial \mathbf{b}_{p}}{\partial \mathbf{F}_{p}}-\mathbf{I}_{p} \otimes \frac{\partial b_{33}}{\partial \mathbf{F}_{p}}\right) \\
& +U_{f}^{\prime}\left(\mathbf{n}_{p} \otimes \mathbf{n}_{p}\right) \otimes \frac{\partial \lambda_{f}}{\partial \mathbf{F}_{p}}+\lambda_{f}\left(\mathbf{n}_{p} \otimes \mathbf{n}_{p}\right) \otimes \frac{\partial U_{f}^{\prime}}{\partial \mathbf{F}_{p}}
\end{aligned}
$$


The Voigt notation form of $\mathbf{C}$ is identified as $\mathbf{C}_{v}$ and given by the components $i_{v}, j_{v}$ as

$$
\begin{aligned}
C_{v_{i_{v} j_{v}}=} & \frac{1}{4}\left(C_{i_{s}\left(i_{v}\right) j_{s}\left(i_{v}\right) k_{s}\left(j_{v}\right) l_{s}\left(j_{v}\right)}+C_{j_{s}\left(i_{v}\right) i_{s}\left(i_{v}\right) k_{s}\left(j_{v}\right) l_{s}\left(j_{v}\right)}\right. \\
& +C_{i_{S}\left(i_{v}\right) j_{s}\left(i_{v}\right) l_{s}\left(j_{v}\right) k_{s}\left(j_{v}\right)}+C_{\left.j_{s}\left(i_{v}\right) i_{s}\left(i_{v}\right) l_{s}\left(j_{v}\right) k_{s}\left(j_{v}\right)\right)}
\end{aligned}
$$

\section{NUMERICAL EXAMPLES}

The thickness of pelvic floor is obtained from Hoyte et al. [28] and it is equal to $6 \mathrm{~mm}$. The transverse shear stiffnesses are $k_{11}=k_{22}=2.982574837 \times 10^{5} \mathrm{MPa}$. The distributed load applied in all elements is IAP $=1.43 \times 10^{-2} \mathrm{MPa}$, which represents the standing cough state [29].

\subsection{Verification}

We will begin with three schematic examples (isometric, isotonic and quick-release), see Figures 4. The square membrane has dimensions $10 \mathrm{~mm}$ along the sides and $1 \mathrm{~mm}$ thickness. It is discretized by $14 \times 14$ triangular shell elements.

In the isometric test, the whole boundary is fixed in directions 1 and 2. The neural excitation of 0.5 is applied and no load is used. The initial conditions are $u(0)=0, \alpha(0)=0$ and $\lambda^{\mathrm{CE}}(0)=$ 0.99594534892. In the isotonic test, the material is stretched along the direction 1 of the fibers but is allowed to deform freely along direction 2 . The boundary nodes in the left side are fixed in the direction 1 and the middle node of this side is also fixed in the direction 2. Then, the membrane is subjected to a traction along direction 1, increasing with time until it reaches the value of $0.6 \mathrm{MPa}$. Thereafter, the applied traction in kept constant and the neural excitation is the same as before and removed $1 \mathrm{~s}$ later. After the initial passive load, the Cauchy stress reaches a value of 0.59 , consistent with a nominal stress of $0.6 \mathrm{MPa}$ multiplied by the quotient between the reference and current cross-section areas. The effect of the activation reduces the muscle length along the direction fiber, and incompressibility implies that the cross-section area grows. Finally, the end of the neural excitation leads the system back to the state it was before the activation started. Similar results (with a distinct model) can be seen in [30].

Finally a quick-release test of the muscle is performed. The membrane is stretched $1 \%$ of the shell dimensions while stimulating to tetanus, then it is released. The initial conditions used are $u(0)=1.0, \alpha(0)=1.0$ and $\lambda^{\mathrm{CE}}(0)=0.976073$.

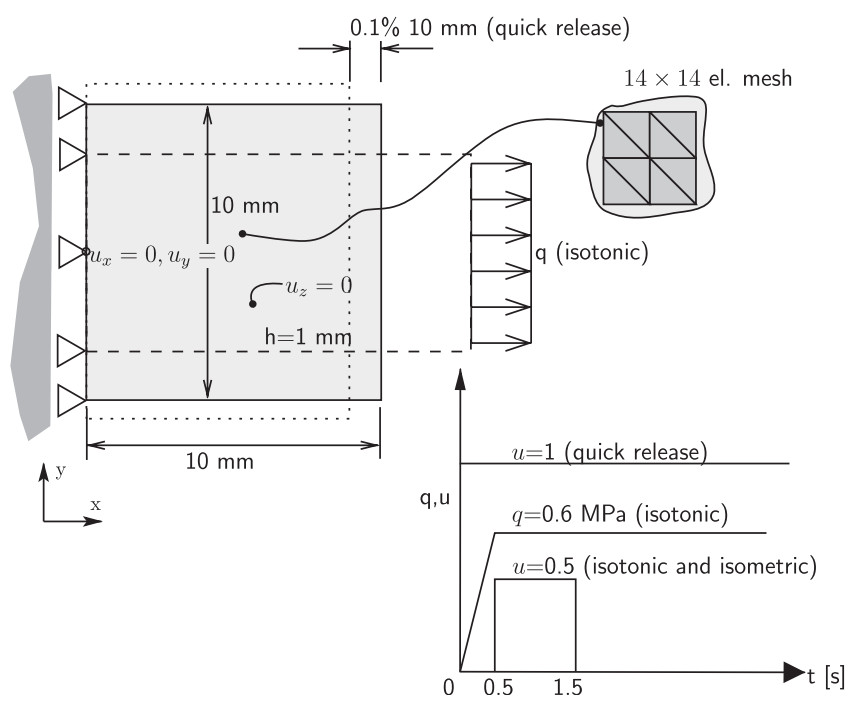

Figure 4. Isometric, isotonic and quick-release tests. 

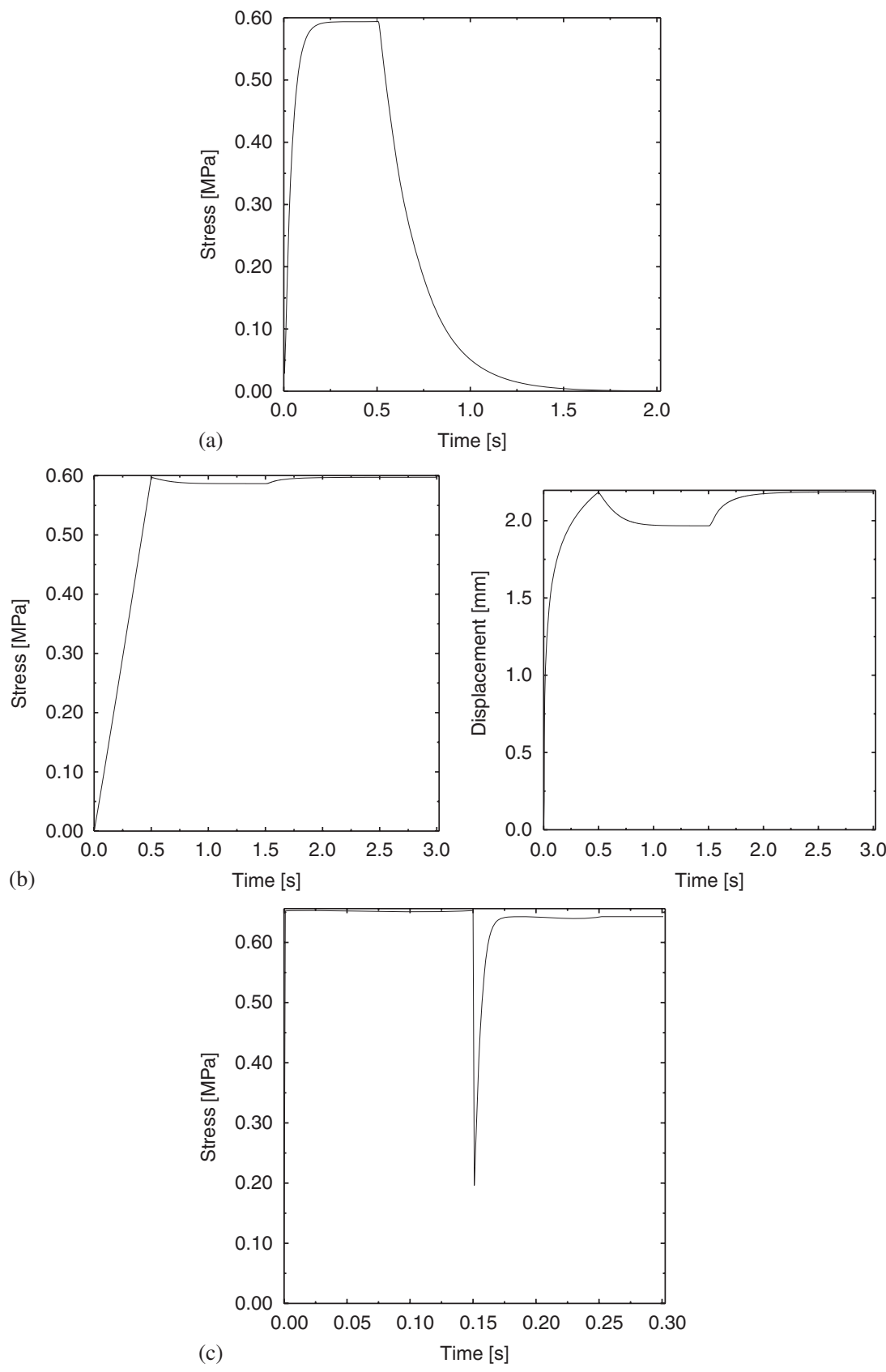

Figure 5. Isometric, isotonic and quick-release test: (a) isometric test; (b) isotonic test; and (c) quick-release test.

The evolutions in time of the Cauchy stress $\sigma_{11}$ and the displacement in direction 1 of the right boundary of the membrane, for the isotonic test, are shown in Figure 5. In the isometric test, the time variation of the corresponding stress has the same shape as the evolution of the activation variable, since the length of the membrane along the fiber direction is constant. In case of the isotonic test, the stress curve increases linearly with time in the half second. For the quick-release test, the Cauchy stress is similar to the isometric test once this test involves the static contraction of the membrane without any visible movement before the stretch. 

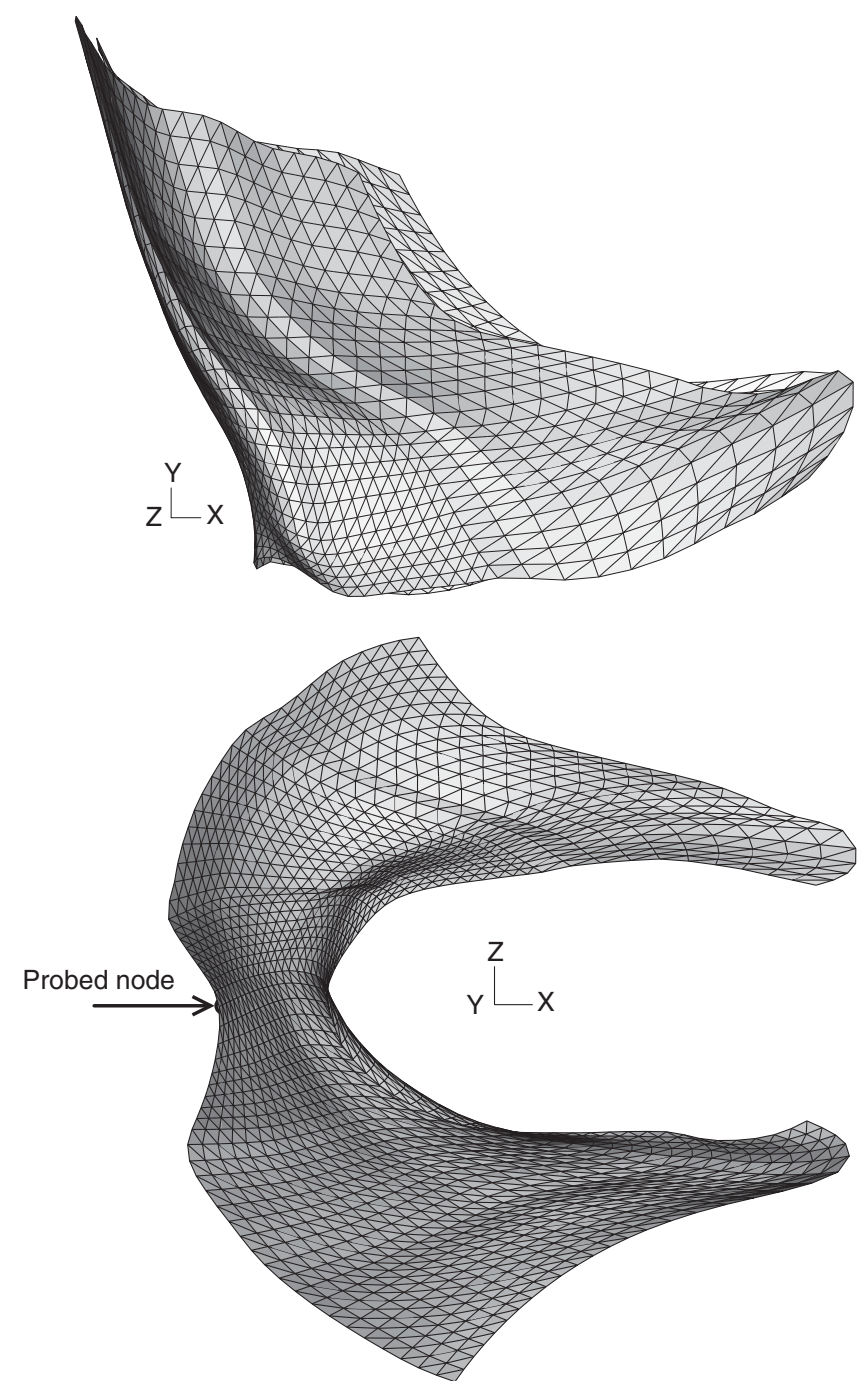

Figure 6. Undeformed mesh obtained from the point-set: two perspectives.

\subsection{Pelvis}

Owing to the thin nature of the levator ani muscle, it is very difficult to construct geometrical models of the pelvic floor from MRI or CT data. Although this has been done successfully in the past for visualization purposes [11], we found these models inadequate for a finite element simulation, since the meshes used were inappropriate. Hence, we opted to use the geometrical point data obtained from cadaver measurements by Janda et al. [31] to reconstruct the surface of the levator ani muscle. All measurements were performed on one embalmed 72 year old female cadaver obtained for scientific research with no known pathologies of the pelvic floor. The result is a 3D point-set of the pelvic floor that is available on the internet. We constructed a surface from this point-set in two consecutive steps. First, the edges of the muscles are defined using splines. Afterward, from these splines, NURBS surfaces are created using the Rhino software. Once triangulated, these surfaces provide a good geometrical model for an FEM simulation. The perspectives $(x-y)$ and $(x-z)$ of the undeformed mesh are shown in Figure 6.

The thickness of pelvic floor is obtained from Hoyte et al. [28] and it is equal to $6 \mathrm{~mm}$. The transverse shear stiffnesses are $k_{11}=k_{22}=2.982574837 \times 10^{5} \mathrm{MPa}$. The distributed load applied in all elements is IAP $=1.43 \times 10^{-2} \mathrm{MPa}$, which represents the standing cough state [29]. While 


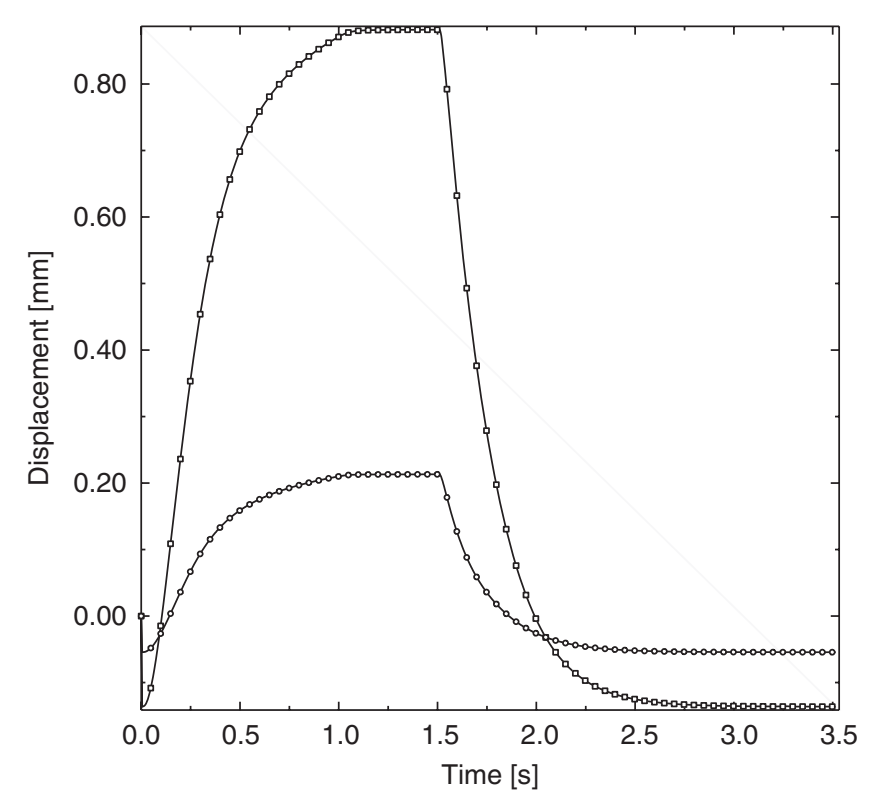

Figure 7. $u_{1}-u_{2}$ diagram for the pelvis.
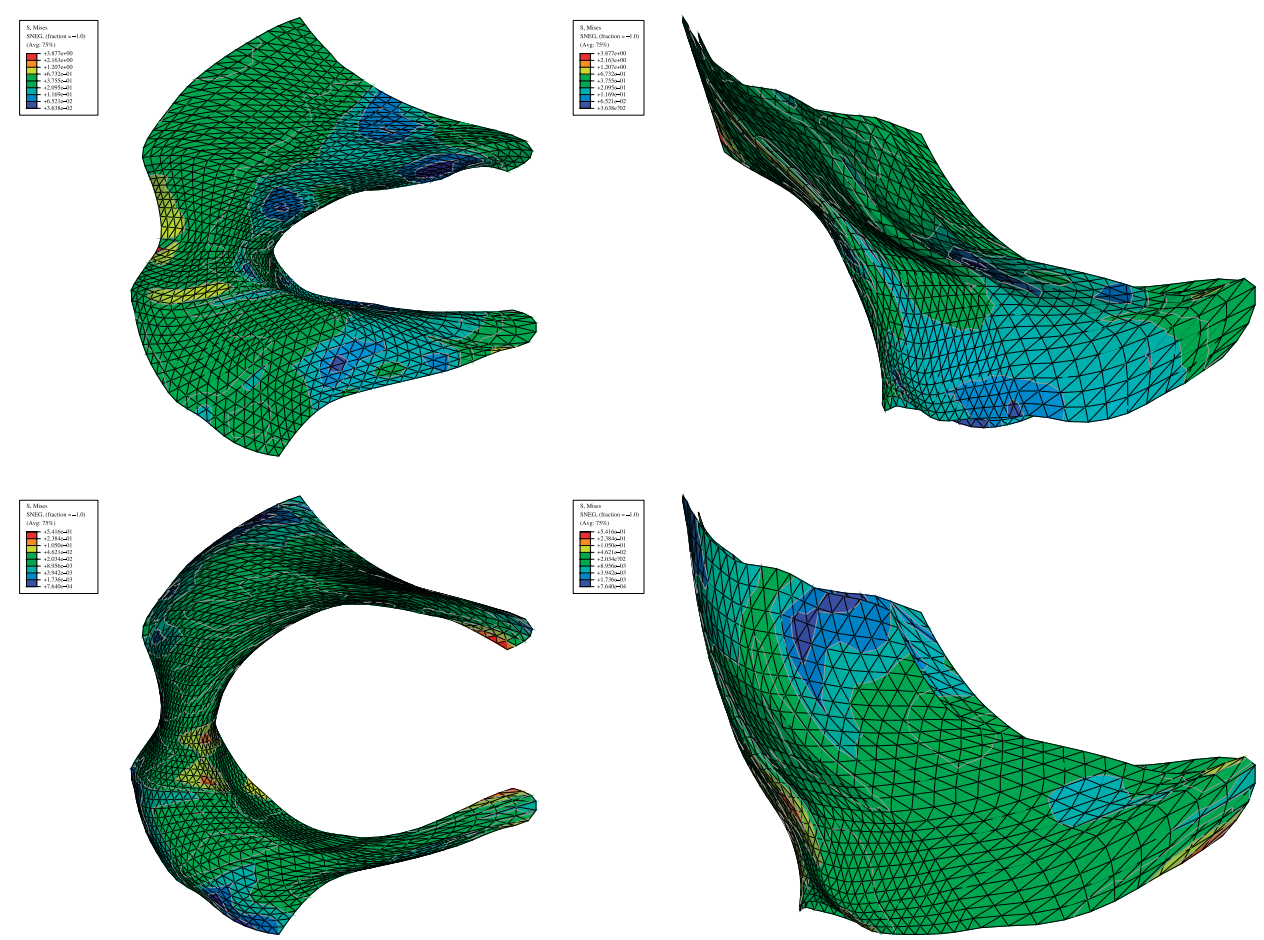

Figure 8. Pelvis simulation: von-Mises stress contour plots for steps 3 and 5.

maintaining the IAP, the muscle is activated for approximately $2 \mathrm{sec}$ with a neural excitation $u(t)=0.5$. After that, the muscle is deactivated. The initial direction of the fibers is chosen to be in the direction of the maximum principal Cauchy stress in the elements when applying only anIAP.

The $u_{1}-u_{2}$ diagram is shown in Figure 7.

The Cauchy stress contour plots for steps 3 and 5 are shown in Figure 8. It is clear that the IAP applied to the inner surface of the levator ani has the expected effect of widening the urogenital 

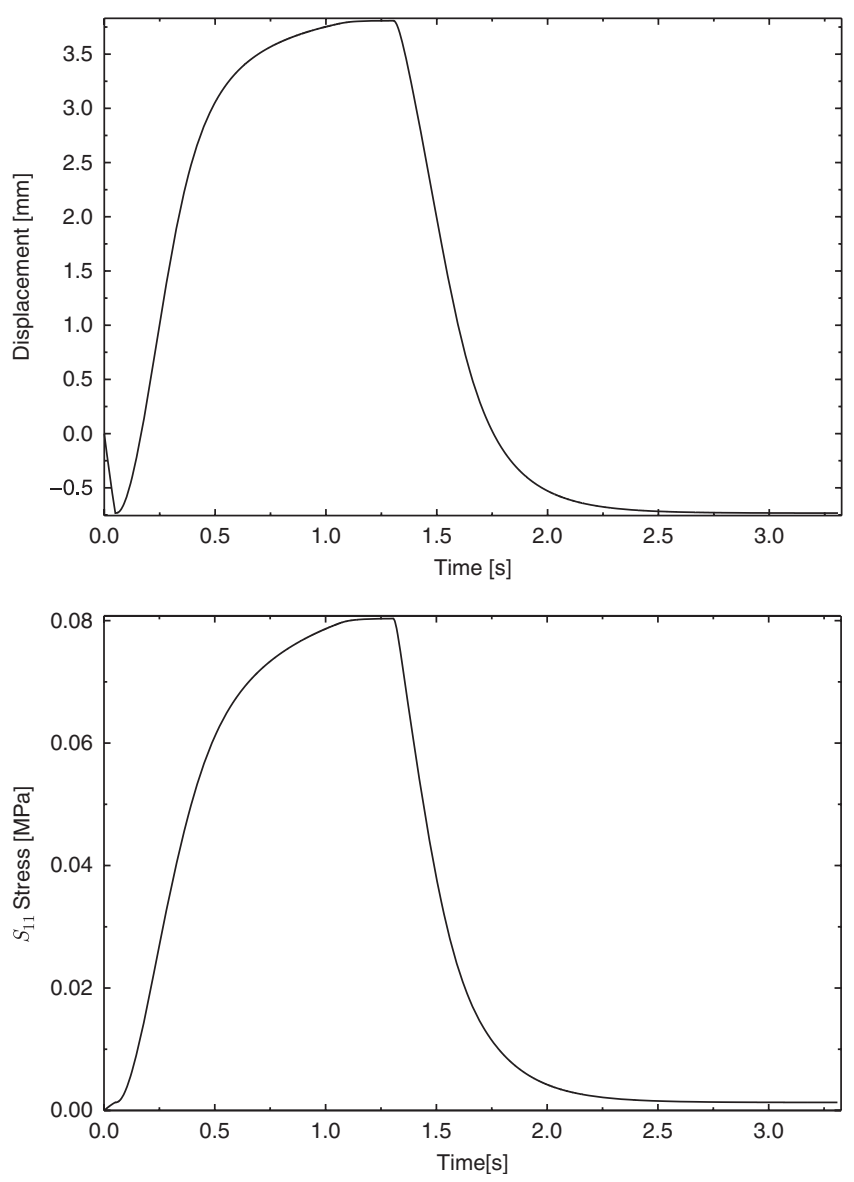

Figure 9. Pelvis $u_{1}$ displacement and $S_{11}$ stress.

hiatus. Figures 8 and 9 show that the muscle activation has the expected opposite effect and also a rising effect. It is also important to observe that when the muscle is passively submitted to an IAP, the regions of maximum stress occur at the sling attachment points of the levator ani, where the most postpartum lesions occur.

\section{CONCLUSIONS}

In this work we developed a new constitutive model and FE implementation of the passive and active behaviors of the skeletal muscles that extend the previous related works in the field $[30,32,33]$. One of the simulations involved passive and active deformations of a muscle of the pelvic floor. The present incorporation in the computational model of the three elements is relevant to model faster contractions, as, for instance, to prevent urinary incontinence in coughing situations.

\section{REFERENCES}

1. Fung YC. Skeletal muscle. Biomechanics Mechanical Properties of Living Tissues (2nd edn), Chapter 9. Springer: Berlin, 1993; 392-425.

2. Hill AV. The heat of shortening and the dynamic constants of muscle. Proceedings of the Royal Society of London, Series B 1938; 129:136-195.

3. Pandy MG, Zajac FE, Sim E, Levine WS. An optimal control model for maximum-heigth human jumping. Journal of Biomechanics 1990; 23(12):1185-1198.

4. Crowningsfield R, Brand R. A physiologically based criterion of muscle force prediction in locomotion. Journal of Biomechanics 1981; I4:793-801. 
5. Seireg A, Arvikar R. Biomechanical Analysis of the Musculoskeletal Structure for Medicine and Sports. Hemisphere: Washington, DC, 1989.

6. Zajac FE, Topp EL, Stevenson PJ. A dimensionless musculotendon model. In Proceedings of the Eighth Annual Conference of IEEE Engineering in Medical and Biology Society, Worth FT (ed.), 1986.

7. Zajac FE. Muscles and tendon: properties, models, scaling and application to biomechanics and motor control. CRC Critical Review Biomedical Engineering 1989; 17:359-411.

8. Pandy MG. Computer modelling and simulation of human movement. Annual Review of Biomedical Engineering 2001; 3:245-273.

9. Audu ML, Davy DT. A dynamic optimization technique for predicting muscle forces in the swing phase of gait. Journal of Biomechanics 1987; 20(2):187-201.

10. Winters JM. Hill-based muscle models: a systems engineering perspective. In Multiple Muscle Systems: Biomechanics and Movement Organization, Winters JM, Woo SL (eds). Springer: Berlin, 1990.

11. Teran J, Blemker S, Ng Thow Hing V, Fedkiw R. Finite volume methods for the simulation of skeletal muscle. Eurographics/SIGGRAPH Symposium on Computer Animation, 2003.

12. Otten E. Inverse and forward dynamics: models of multi-body systems. Philosophical Transactions of the Royal Society of London, Series B 2003; 358:1493-1500.

13. Fernandez JW, Hunter PJ. An anatomically based patient-specific finite element model of patella articulation: towards a diagnostic tool. Biomechanics and Modelling in Mechanobiology 2005; 4(1):20-38.

14. Fernandez JW, Ho A, Walt S, Anderson IA, Hunter PJ. A cerebral palsy assessment tool using anatomically based geometries and free-form deformation. Biomechanics and Modelling in Mechanobiology 2005; 4(1):39-56.

15. Fernandez JW, Pandy MG. Integrating modelling and experiments to assess dynamic musculoskeletal function. Experimental Physiology 2006; 91(2):371-382. DOI: 10.1113/expphysiol.2005.031047.

16. Dong F, Clapworthy GJ, Krokos MA, Yao J. An anatomy-based approach to human muscle modeling and deformation. IEEE Transactions on Visualization and Computer Graphics 2002; 8(2):233-242.

17. Van Loocke M, Lyons CG, Simms CK. A validated model of passive muscle in compression. Journal of Biomechanics 2006; 39:S36-S37.

18. Grieve AP, Armstrong CG. Compressive properties of heft tissues. Biomechanics XI-A, International Series on Biomechanics, vol. 53. Free University Press: Amsterdam, 1988; 1-536.

19. Bosboom EMH, Hesselink MKC, Oomens CWJ, Bouten CVC, Drost MR, Baaijens FPT. Passive transverse mechanical properties of skeletal muscle under in vivo compression. Journal of Biomechanics 2001; 34:1365-1368.

20. Otten E, Hulliger M. A finite elements approach to the study of functional architecture in skeletal muscle. Zoology Analysis of Complex Systems 1994; 98:233-242.

21. van Leuween JL, Kier WM. Functional design of tentacles in squid: linking sarcomere ultrastruture to gross morphological dynamics. Phisiology Transactions of the Royal Society of London, Series B 1997; 352:551-571.

22. Humphrey JD, Strumpf RK, Yin FCP. Determination of a constitutive relation for passive myocardium. I. A new functional form. Journal Biomechanics Engineering (ASME) 1990; 112:333-339.

23. Spencer AJM. Constitutive theory for strongly anisotropic solids. In Continnum Theory of the Mechanics of Fiber-reinforced Composites, Spencer AJM (ed.). Springer: New York, 1984; 1-32.

24. Antman SS, Schuricht F. Incompressibility in rod and shell theories. Mathematical Modelling and Numerical Analysis 1999; 33:289-304.

25. Humphrey JD, Yin FCP. On constitutive relations and finite deformations of passive cardiac tissue: I. A pseudostrain-energy function. Journal of Biomechanical Engineering (ASME) 1987; 109:298-304.

26. Böl M, Reese S. A new approach for the simulation of skeletal muscles using the tool of statistical mechanics. Materialwissenschaft und Werkstofftechnik 2007; 38(12):955-964.

27. McLean SG, Su A, van der Bogert AJ. Development and validation of a 3-d model to predict knee joint loading during dynamic movement. Journal of Biomechanical Engineering (ASME) 2003; 125:864-874.

28. Hoyte L, Jakab M, Warfield SK, Shott S, Flesh G, Fielding JR. Levator ani thickness variations in symptomatic and asymptomatic women using magnetic resonance-based 3-dimensional color mapping. American Journal of Obstetrics and Gynecology 2004; 191:856-861.

29. Cobb WS, Burns JM, Kercher KW, Matthews BD, Norton HJ, Heniford BT. Levator ani thickness variations in symptomatic and asymptomatic women using magnetic resonance-based 3-dimensional color mapping. Journal of Surgical Research 2005; 129:231-235.

30. Martins JAC, Pato MPM, Pires EB. A finite element model of skeletal muscles. Virtual and Physical Prototyping 2006; 1(3):159-170.

31. Janda S, van der Helm FCT, de Blok SB. Measuring morphological parameters of the pelvic floor for finite element modelling purposes. Journal of Biomechanics 2003; 36:749-757.

32. Martins JAC, Pires EB, Salvado R, Dinis PB. A numerical model of passive and active behavior of skeletal muscles. Computer Methods in Applied Mechanics and Engineering 1998; 151:419-433.

33. d'Aulignac D, Martins JAC, Pires EB, Mascarenhas T, Natal Jorge RM. A shell finite element model of the pelvic floor muscles. Computer Methods in Biomechanics and Biomedical Engineering 2005; 8(5):339-347. 